\title{
Adipose Tissue-Derived Mesenchymal Stem Cells (ADMSCs) and ADMSC-Derived Secretome Expedited Wound Healing in a Rodent Model - A Preliminary Study
}

\author{
Hui Ma iD ${ }^{1,2}$ \\ Ping Kuen Lam (iD ${ }^{3}$ \\ Wing Sum Siu ${ }^{1,2}$ \\ Cindy See Wai Tong ${ }^{3}$ \\ Kin Ki Yan Lo iD ${ }^{3}$ \\ Chi Man Koon ${ }^{1,2}$ \\ Xiao Xiao $\mathrm{Wu}^{1,2}$ \\ Xiang $\mathrm{Li}^{1,2}$ \\ Wen Cheng ${ }^{1,2}$ \\ Wai Ting Shum ${ }^{1,2}$ \\ Ping Chung Leung ${ }^{1,2}$ \\ 'Institute of Chinese Medicine, The \\ Chinese University of Hong Kong, Shatin, \\ Hong Kong, Special Administrative \\ Region of China; ${ }^{2}$ State Key Laboratory \\ of Research on Bioactivities and Clinical \\ Applications of Medicinal Plants, The \\ Chinese University of Hong Kong, Shatin, \\ Hong Kong, Special Administrative \\ Region of China; ${ }^{3}$ Chow Tai Fook-Cheng \\ Yu Tung Surgical Stem Cell Research \\ Center, Department of Surgery, The \\ Chinese University of Hong Kong, Shatin, \\ Hong Kong, Special Administrative \\ Region of China
}

Correspondence: Ping Chung Leung Institute of Chinese Medicine, The

Chinese University of Hong Kong, Shatin, Hong Kong, Special Administrative Region of China

Tel +85222528868

Email pingcleung@cuhk.edu.hk
Introduction: The biological role of mesenchymal stem cells (MSCs) in wound healing has been demonstrated. However, there were limited studies on the healing effect of secretome which consists of many biological factors secreted by MSCs. In this study, we aimed to compare the therapeutic effects of secretome with MSCs on facilitating wound healing.

Methods: Green fluorescent protein labelled adipose-derived MSCs (GFP-ADMSCs) or secretome was injected in the full-thickness skin excision model on SD rats. The wound healing process was evaluated by calculating the healing rate and the histological examinations on skin biopsy. The cell viability, proliferation and mobility of the rat dermal fibroblasts were compared after different treatments. The inflammatory response in macrophages was indicated by the level of nitric oxide (NO) and inflammatory cytokines through NO assay and ELISA.

Results: On day 5 and day 14, both MSCs and secretome accelerated the wound healing, secretome further enhanced the process. GFP-MSCs were detected 10 days after transplantation. The level of IL-6 and TNF- $\alpha$ in blood was reduced after MSCs and secretome treatments. The expressions of VEGF and PCNA were increased after treatment, higher intensity of VEGF was observed in secretome-injected tissue. The concentrations of total protein and VEGF in secretome were $2.2 \pm 0.5 \mathrm{mg} / \mathrm{mL}$ and $882.0 \pm 72.7 \mathrm{pg} / \mathrm{mL}$, respectively. The cell viability and proliferation of FR were promoted significantly after the treatment. The scratch test showed that secretome accelerated the wound healing speed. Secretome reduced the metabolism of macrophages remarkably, but it did not decrease the level of macrophagesecreted NO. The expression of the pro-inflammatory cytokines (IL-6, MCP-1 and TNF- $\alpha$ ) was downregulated significantly.

Conclusion: Our study indicated both MSCs and MSCs-derived secretome enhanced the wound healing process in early phase. Secretome further promoted the healing effects through promoting the fibroblast proliferation and migration and suppressing the inflammatory response.

Keywords: MSCs, secretome, wound healing, local injection, skin

\section{Introduction}

Skin is the largest organ, which represents $8 \%$ of the body mass. ${ }^{1}$ It is critical in protecting against foreign pathogens, regulating body temperature and water balance. $^{2}$ The interruption of normal anatomic structure and function of the skin can be caused by burn, cut or puncture. Wound healing involves complex 
and well-coordinated cascades including haemostasis (cell migration), inflammation, proliferation and remodelling. ${ }^{3}$ The process of wound healing can be compromised by both local and systemic factors, such as infection, ischemia and systemic chronic diseases (eg, diabetes), resulting in chronic and refractory ulcers. ${ }^{2,3}$ It causes a huge burden to the health care system. There are 8.2 million people suffering from wound with or without infections. ${ }^{4}$ The cost of wound treatment ranges from 28.1 billion to 96.8 billion US dollars due to the increase in health care, an aging population and the increase in diabetic population. ${ }^{5}$ Current treatments for wound healing include: (i) growth factors and cytokines, such as vascular epithelial growth factor (VEGF), platelet-derived growth factor (PDGF) and beta fibroblast growth factor $(\beta \mathrm{FGF})$; (ii) skin substitutes, which are generated by tissue engineering and commercialized, such as Integra $^{\mathrm{TM}}$ (Johnson \& Johnson), Alloderm ${ }^{\circledR}$ (Biohorizons) and Epicel ${ }^{\mathrm{TM}}$ (Vericel Corporation); (iii) hyperbaric oxygen therapy $\left(\mathrm{HBO}_{2}\right)$ and (iv) skin graft. ${ }^{6-}$ 8 However, these conventional treatments are not effective.

Regenerative medicine using stem cells provides a novel tool for the treatment of non-healing ulcers. Mesenchymal stem cells (MSCs) have the properties of self-renewal and differentiating into multiple cell lineages, including osteoblasts, chondroblasts and adipoblasts. ${ }^{9}$ The therapeutic effects of MSCs have been studied in various wound models, including acute excisional wounds, diabetic skin ulcer, radiation and burn wounds. ${ }^{10-12}$ However, short cell survival time in the recipient wounds limits the translation of MSCs from bench to clinic.

It is believed that the paracrine activity is the main underlying mechanism of the MSCs-based cell therapy. They are able to secrete a group of soluble factors, including growth factors, cytokine and chemokines to the extracellular matrix. These factors modulate multiple physiological processes such as inflammation, apoptosis and angiogenesis. Tumour growth factor- $\beta$ (TGF- $\beta$ ), VEGF, keratinocyte growth factor (KGF), FGF2, PDGF, fibronectin and collagen have been found in the supernatant of MSCs (secretome), and they accelerated the process of wound healing. ${ }^{13}$
In this study, we aimed to investigate the therapeutic effects of the locally injected MSCs and secretome in both in vitro and in vivo acute excisional wound model on SD rats.

\section{Materials and Methods}

All surgical activities were performed regarding the Animals (Control of Experiments) Ordinance Chapter 340, Department of Health, Hong Kong. And the study was approved by the Animal Experimentation Ethics Committee of the Chinese University of Hong Kong.

\section{ADMSCs Cultivation and Identification}

According to the criteria from the International Society for Cell and Gene Therapy (ISCT), the surface marker (CD29, CD45, CD90) and tri-lineage differentiation potential were identified. ${ }^{14}$ ADMSCs were cultured in low glucose Dulbecco's Modified Eagle Medium (DMEM) (Gibco, HK) and characterized as previously described. ${ }^{14}$ Briefly, the cells in $\mathrm{P} 2$ were cultured to $80 \%$ confluency before they were used for the identification. Male transgenic SD rats (300-350g) which expresses green fluorescent protein (GFP) (SDTg (CAG-EGFP) CZ-0040sb; SLC Inc, Shizuoka, Japan) were used as donor. After cultivation, ADMSCs were labelled with phycoerythrin-conjugated antibodies against CD29, CD45, and CD90 (Abcam Inc., Cambridge, UK). ${ }^{15}$ To identify the differentiation potential, ADMSCs were cultured in adipogenic, chondrogenic, and osteogenic differentiation culture media according to the manufacturer's protocols (Invitrogen, LifeTechnologies $^{\mathrm{TM}}$, HK). The differentiated adipocytes were stained with Oil Red O, chondrocytes with Alcian Blue, and osteocytes with Alizarin Red S stain to identify intracytoplasmic lipid, extracellular glycosaminoglycan, and calcium deposits, respectively. All chemicals were purchased from Sigma-Aldrich, Shanghai, China. ${ }^{15}$

\section{Secretome Collection}

ADMSCs in P2 were cultured to reach $80 \%$ confluency. Then they were cultured in serum-free DMEM for 18 hours prior to the secretome collection. The culture medium was collected into a $50 \mathrm{~mL}$ centrifuge tube and centrifuged at $1500 \mathrm{rpm}$ for 10 minutes to remove 
cell debris. Then the centrifuged cell supernatant was transferred into centrifugal filter units with a $3 \mathrm{kDa}$ molecular mass cut-off (Amicon ${ }^{\circledR}$ Ultra-15, Millipore) and concentrated to 50-fold. The centrifuged cell supernatant was centrifuged for 45 minutes at a speed of $4000 \mathrm{rcf}$. The concentrated solution was aliquoted into $1.5 \mathrm{~mL}$ tubes (Eppendorf, $\mathrm{HK}$ ) and stored in $-80^{\circ} \mathrm{C}$ for further use. The secretome used in the study was collected from one batch experiment.

\section{Total Protein Content: Bicinchoninic Acid (BCA) Assay}

Bovine serum albumin (BSA) was used as a standard to calculate the protein concentration in the secretome. The BCA assay was made from two solutions: $4 \%$ copper sulphate and the Bicinchoninic acid (BCA) solution from Sigma Life Science, USA. The optical absorbance was measured at $562 \mathrm{~nm}$ with a microplate reader. The protein concentration of secretome was calibrated through the plot of standard BSA concentrations. Three samples were used for the test. Each sample was tested in triplicate.

\section{VEGF Concentration: Enzyme-Linked Immunosorbent Assay (ELISA)}

Concentration of VEGF released by ADMSCs was determined by Quantikine ${ }^{\circledR}$ ELISA kit (R\&D System, Inc., Minneapolis, USA). All procedures were performed according to the manufacturer's instructions. DMEM was used as negative control. Three samples were used for the test. Each sample was tested in triplicate.

\section{In vivo Study} Wound Model

Adult female SD rats weighed 300-350 g were used for the experiment. They were anesthetized with the cocktail of ketamine $(50 \mathrm{mg} / \mathrm{kg})$ and xylazine $(10 \mathrm{mg} / \mathrm{kg})$ through intra-peritoneal injection. During the surgery, their body temperature was maintained at $37^{\circ} \mathrm{C}$ by a warm pad. A $2.5 \times 2.5 \mathrm{~cm}$ full-thickness skin excision was induced on the dorsal area of the rat using scissors and forceps. Total 4 million MSCs $(n=15) / 125 \mu \mathrm{L}$ secretome $(\mathrm{n}=15)$ was injected at five sites (four surrounding the wound $1 \mathrm{~mm}$ to the edge and one in the middle) using insulin syringe. No treatment was given to the control animals $(n=15)$. After surgery, the wound was covered by sterile Vaseline gauze and all animals were kept in cage individually. Vaseline gauze was changed every three days until ten days.

\section{Wound Healing Evaluation: Healing Rate}

After surgery, the wound size was measured on day 5, 14, 21,28 . The healing rate was calculated as:

(day 0 - day $5 / 14 / 21 / 28$ )/day $0 * 100$

\section{Microscopic Examinations}

In each group, five animals were sacrificed on day 5 and day 14, respectively, after surgery. Paraffin embedded tissue was used for the histological examinations. Five photos were taken under various magnifications (100x, 200x for GFP staining, 200x for VEGF staining, 400x for PCNA staining) at the wound area for examination.

To identify the transplanted GFP-MSCs, immunohistochemical (IHC) staining was performed on hydrated paraffin sections using anti-GFP antibody (Abcam, Cambridge, MA, USA).

Mouse monoclonal antibody against VEGF (Santa Cruz, Dallas, TX, USA), and proliferating cell nuclear antigen (PCNA) (Abcam, Cambridge, MA, USA) were used in IHC staining to detect growth factor release and cell proliferation.

\section{Elisa}

Blood samples were taken on day 5 and day 14 after the treatments. The level of IL- 6 and TNF- $\alpha$ was examined by ELISA kits (BD OptEIA ${ }^{\mathrm{TM}}$, BD Biosciences, San Diego, CA, USA). All procedures were performed according to the manufacturer's instructions.

\section{In vitro Study}

Rat dermal fibroblast cell line (FR, CRL-1213, ATCC, Manassas, VA) and macrophages (RAW 264.7, TIB-71, ATCC, Manassas, VA) were used for the in vitro studies.

\section{Cell Viability/Cytotoxicity Assay}

After treated with secretome in different times of dilution $(1,1 / 5,1 / 10,1 / 25,1 / 50,1 / 100,1 / 250,1 / 500,1 /$ 1000), the cell viability of FR and RAW 264.7 were tested by the 3-[4,5-dimethylthiazol-2-yl]-2,5-diphenyltetrazolium bromide (MTT) (Sigma, USA) assay. The cells were seeded in 96-well plates, respectively (FR: 

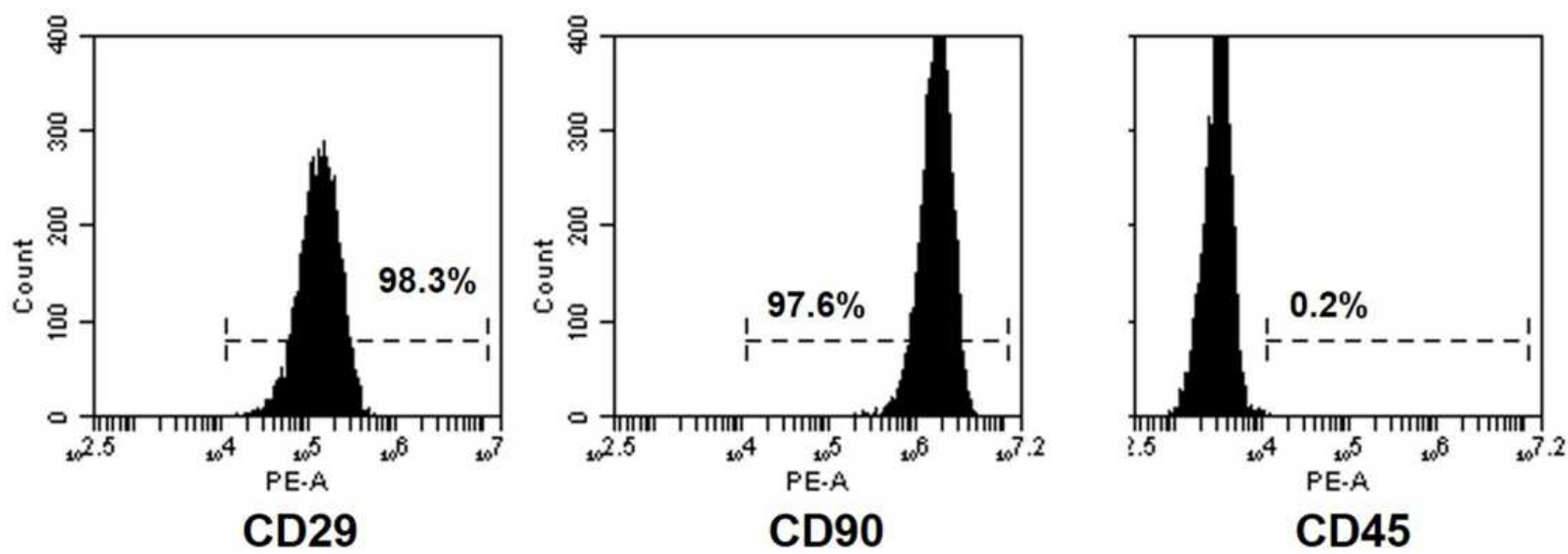

Figure I Flow cytometry results for MSCs. The MSCs were identified to be positive for CD29 (98.3\%) and CD90 (97.6\%), negative for CD45 (0.2\%).

$5 \times 10^{3} /$ well; RAW 264.7: $1 \times 10^{5} /$ well) for 24 hours. Secretome was added and incubated for another 24 hours. On the third day, the cell viability was measured by the reduction of MTT dye in living cells to blue formazan crystals at optical density at $540 \mathrm{~nm}$. Cells cultured in DMEM were used as control.

\section{Cell Proliferation Assay (BrdU)}

BrdU proliferation assay was used to detect FR proliferation after treated by secretome in different times of dilution $(1,1 / 5,1 / 10,1 / 25,1 / 50,1 / 100,1 / 250,1 / 500,1 / 1000)$ for 24 hours. All procedures were performed according to the manufacturer's instructions (Sigma, USA). Cells were incubated with BrdU-labelling solution for 4 hours. After being fixed and denatured by FixDenat, cells were incubated with anti-BrdU antibody for 90 minutes. Substrate solution was used for colour reaction and the optical density was measured at $450 \mathrm{~nm}$. Cells cultured in DMEM were used as control. Medium without cells was used as blank control.

\section{Scratch Test}

The migration of FR was examined using scratch test. $5 \times 10^{4}$ of FR were seeded in each well of 24-well plate supplied with low glucose DMEM, 10\% FBS (Gibco, USA) and 1\% penicillin-streptomycin (PS) (Invitrogen Co., Carlsbad, CA, USA). After the cell reached high confluency, the medium was replaced with DMEM containing $1 \%$ FBS, and the cells were starved for 24 hours. Then, two crosses were scrapped in each well using $200 \mu \mathrm{L}$ tips. Two photos of the two crosses were taken under microscope at $4 \mathrm{x}$ magnification, secretome in different times of dilution $(1,1 / 5,1 / 10,1 / 25,1 /$ $50,1 / 100,1 / 250,1 / 500,1 / 1000)$ was added to each well.
Normal DMEM was used as control. The images were analysed by TScratch software. The percentage of closed area was compared with the area before treatment and shown as relative wound area.

\section{Nitric Oxide (NO) Inhibitory Assay}

Lipopolysaccharides (LPS) was used to induce inflammatory response in RAW 264.7 (macrophages), the severity was shown by the concentration of NO. RAW 264.7 were seeded at the density of $4 \times 10^{5} /$ well in a 24 -well plate for overnight incubation. Then, secretome in different times of dilution $(1,1 / 5,1 / 10,1 / 25,1 / 50,1 / 100,1 / 250,1 / 500,1 /$ 1000) with $1 \mu \mathrm{g} / \mathrm{mL}$ LPS was added to each well and incubated for 24 hours. After incubation, $100 \mu \mathrm{L}$ cell supernatant was transferred to a 96-well plate. $100 \mu \mathrm{L}$ Griess Reagent was added to each well. After 10 minutes, the plate was read at $540 \mathrm{~nm}$ in a microplate reader. The standard curve was plotted by the given concentration of sodium nitric $\left(\mathrm{NaNO}_{3}\right)$. DMEM was used as negative control.

\section{Elisa}

The concentrations of pro-inflammatory cytokines IL-6, MCP-1, TNF- $\alpha$ in RAW 264.7 supernatant were measured using ELISA kits (BD OptEIA ${ }^{\mathrm{TM}}$, BD Biosciences, San Diego, CA, USA). All procedures were performed according to the manufacturer's instructions.

\section{Statistical Analysis}

All data are expressed in mean \pm standard error of the mean (SEM). All data underwent one-way or two-way analysis of variance (ANOVA) using GraphPad Prism 5. A $p$ value less than 0.05 was considered as significant. Dunnett test was applied as post-hoc comparison. 
Table I Total Protein Content and VEGF Concentration of Three Samples. Three Samples from Same Bench of Secretome Were Used for the Tests. Mean Total Protein and VEGF Concentration of the Secretome Used for the Study Was Shown. No Significant Difference Was Observed Among the Three Samples

\begin{tabular}{|l|c|c|}
\hline Sample & $\begin{array}{c}\text { Total Protein Content } \\
(\mathbf{m g} / \mathbf{m L})\end{array}$ & $\begin{array}{c}\text { VEGF Concentration } \\
\mathbf{( p g / m L})\end{array}$ \\
\hline $\mathrm{I}$ & $2.64 \pm 0.13$ & $954.76 \pm 19.45$ \\
2 & $1.74 \pm 0.13$ & $809.34 \pm 29.46$ \\
3 & $2.14 \pm 0.13$ & $881.84 \pm 27.11$ \\
Mean & $2.17 \pm 0.45$ & $881.98 \pm 72.71$ \\
\hline
\end{tabular}

\section{Results}

\section{ADMSCs Expressed Surface Marker: CD29, CD90, and Indicated Tri-Lineage Differentiation Potential}

ADMSCs expressed CD29 and CD45, but negative in CD45 (Figure 1). They also showed the differentiation potential into adipocytes, chondrocytes and osteocytes (Supplemental Figure 1). ${ }^{15}$

No significant difference was observed in the total protein and VEGF concentration among secretome samples.

The total protein content and VEGF concentration of the three secretome samples were tested using BCA array and ELISA. The respective concentration was $2.14 \pm 0.13 \mathrm{mg} / \mathrm{mL}$ and $881.98 \pm 72.71 \mathrm{pg} / \mathrm{mL}$ (mean \pm SD). No significant difference was observed among the three samples $(\mathrm{p}>0.05)$ (Table 1).

\section{In vivo Study}

MSCs and Secretome Accelerated the Healing Rate

On day 5 and day 14, both MSCs and secretome accelerated the wound healing significantly $(\mathrm{p}<$ 0.05), compared with MSCs, secretome further promote the effect $(\mathrm{p}<0.05)$. No significant change was observed among the three groups on day 21 and day 28 (Figure 2A). The wound size is shown in Table 2. The wound healing process completed after 28 days (Figure 2B).

\section{VEGF Expression and Number of PCNA Positive Cells Were Increased}

IHC staining showed GFP-positive cells were present at the injection sites up to day 10 (Figure $3 \mathrm{~A}$ and $\mathrm{B}$ ).
No GFP-positive cells were detected afterwards. Both MSCs and secretome increased the deposition of VEGF in the recipient wounds (Figure 3C). More VEGF was deposited in the secretome-treatment group (Figure 3C). Anti-PCNA staining was introduced to show the level of cell proliferation. Cell proliferation was promoted significantly after secretome injection on both day 5 and day 14 (Figure 3D). The number of PCNA positive cells was the highest in secretome group $(\mathrm{p}<0.05)$. On day 5 and day 14 , the number of proliferated cells decreased slightly after the MSCs injection (Figure 3E).

\section{MSCs and Secretome Reduced the Release of IL-6 and TNF- $\alpha$}

Both MSCs and secretome reduced the level of proinflammatory cytokines (IL-6 and TNF- $\alpha$ ) on day 5 and day 14 after treatment (MSCs vs control: $\mathrm{p}<0.01$; secretome vs control: $\mathrm{p}<0.001)$. Compared with MSCs, secretome further suppressed the expressions of the proinflammatory cytokines $(p<0.05)$ (Figure 4A, B).

\section{In vitro Study}

Diluted Secretome Enhanced the Viability, Proliferation and Migration of Dermal Fibroblasts (FR)

The results indicated that the concentrated secretome was toxic to the metabolism of FR $(p<0.001)$. At a broad range of dilution $(1 / 10-1 / 1000)$, secretome enhanced cell viability significantly $(\mathrm{p}<0.05)$ (Figure $5 \mathrm{~A})$.

The optical density read at $450 \mathrm{~nm}$ wavelength is shown in Figure 5B. Higher absorbance indicated more proliferation. As shown in the figure, the concentrated secretome showed no effects on FR proliferation. After diluted into $1 / 5,1 / 10,1 / 25,1 / 50,1 / 100,1 / 250$ and $1 /$ 500 , it promoted FR proliferation significantly $(\mathrm{p}<$ 0.05) (Figure 5B).

Healing effects of secretome on FR were tested by scratch assay. As shown in Figure 5C, same open wound was scratched by a pipette tip in the culture well. After 18 hours, cells treated with the secretome migrated at the lowest velocity, which was similar with the control. At the relative high concentration $(1 / 5,1 / 10,1 / 25,1 / 50)$, FR migrated significantly faster. The percentage of the closed wound area compared with the original wound area is shown in Figure 5D $(\mathrm{p}<0.05)$. 
A

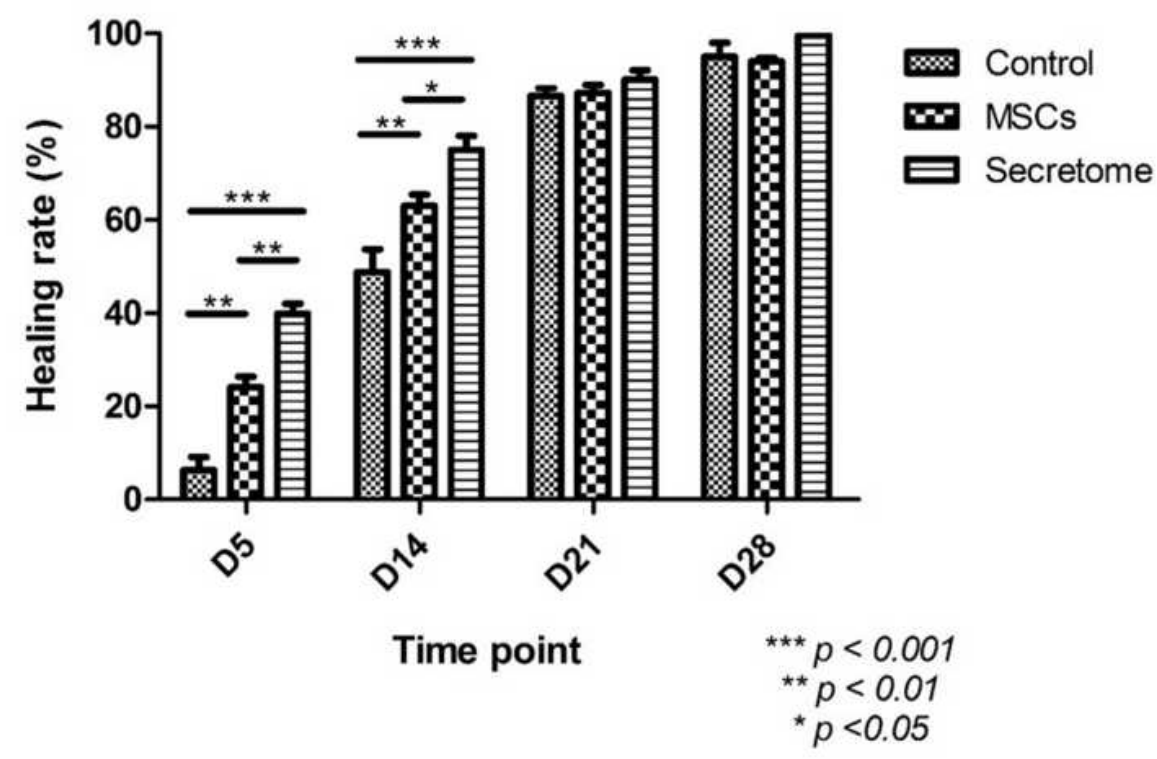

B

Control

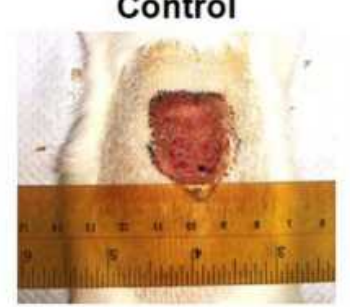

Day 5

Day 14
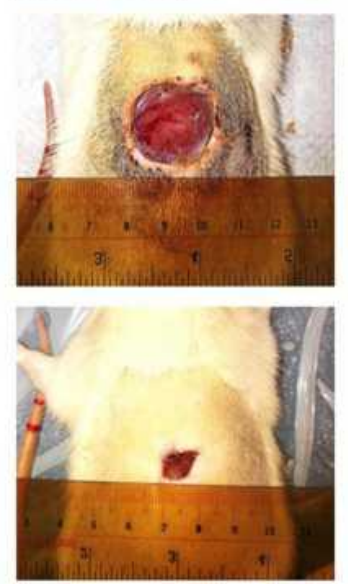

Day 21

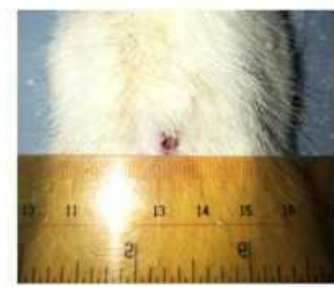

MSCs
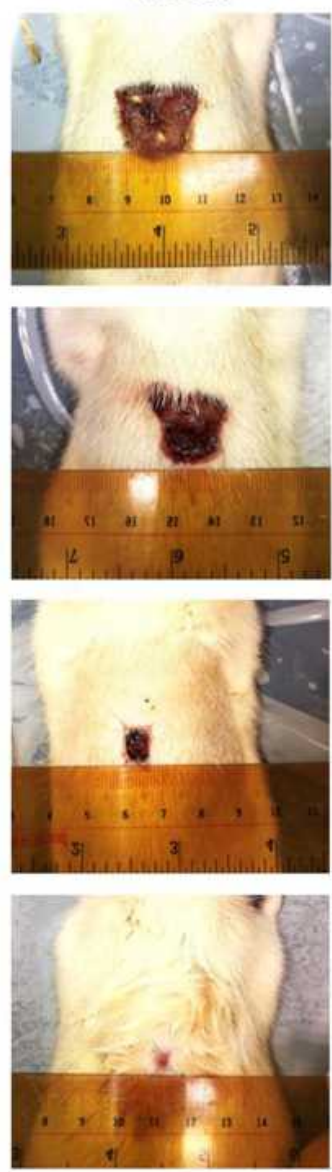

Secretome


Figure 2 Healing rate and photos of wound at different time points. (A) The percentage of wound closure. Both MSCs and secretome promoted wound closure on day 5 and day 14 (day 5: MSCs, ${ }^{* *} \mathrm{p}<0.01$; secretome, ${ }^{* * *} \mathrm{p}<0.001$; day 14: MSCs, ${ }^{* *} \mathrm{p}<0.01$; secretome, ${ }^{* * *} \mathrm{p}<0.001$ ). Compared with MSCs, secretome further accelerated the healing process (day 5: **p $<0.01$; day 14: ${ }^{*} \mathrm{p}<0.05$ ). (B) Photos of wound at different time points. MSCs and secretome reduced the size of wound from day 5 to day 14. The wound was healed in secretome group after 28 days. 
Table 2 Mean Wound Size at Different Time Points. On Day 5 and Day 14, Both MSCs and Secretome Reduced the Wound Size $(p<0.05$ Vs Control). Secretome Further Accelerated the Wound Closure ( $p<0.05$ Vs MSCs)

\begin{tabular}{|l|c|c|c|}
\hline \multirow{2}{*}{ Time } & \multicolumn{3}{|c|}{ Mean Size $\left(\mathbf{c m}^{2}\right)$} \\
\cline { 2 - 4 } & Control & MSCs & Secretome \\
\hline Day 5 & 6.75 & 4 & 3 \\
Day 14 & 2.25 & $\mathrm{I}$ & 0.75 \\
Day 21 & 0.75 & 0.8 & 0.5 \\
Day 28 & 0.45 & 0.3 & 0 \\
\hline
\end{tabular}

Low Dose Secretome Indicated No Cytotoxicity and Reduced the Level of LPS-Induced NO and ProInflammatory Cytokines (IL-6, TNF- $\alpha$ )

After the incubation with secretome for 24 hours, MTT was used to detect the impact on cell metabolism of RAW 264.7. The results indicated that the cell viability was suppressed significantly by the concentrated secretome $(1,1 / 5,1 / 10,1 / 25)(\mathrm{p}<0.05)$. No toxic effect was observed in the cells treated with low concentration secretome $(1 / 50-1 / 1000)$ (Figure 6A).

NO was released by macrophages after LPS induction. The level of NO was correlated with the inflammatory response. Since the highly concentrated secretome presented the cytotoxic effect on the cells, only low concentrations $(1 / 50-1 / 1000)$ were tested. Only slight reduction of NO level was observed without statistical significance (Figure 6B).

After LPS induction, the cell supernatant treated with low-concentrated secretome $(1 / 50-1 / 1000)$ was used for ELISA. The concentrations of the proinflammatory cytokines IL-6, MCP- 1 and TNF- $\alpha$ were examined. The secretion of IL-6 was suppressed remarkably when treated by $1 / 50,1 / 100,1 / 250$, and $1 / 500$ secretome $(\mathrm{p}<0.05$ vs LPS). No difference was found in the supernatant treated by $1 / 1000$ secretome (Figure 6C). The level of MCP-1 was reduced significantly after incubation with various concentrations of secretome $(1 / 50-1 / 1000)(\mathrm{p}<0.05$ vs LPS) (Figure 6D). As shown in Figure 6E, the expression of TNF- $\alpha$ was decreased after the secretome $(1 / 50,1 / 100$, $1 / 250,1 / 500)$ treatment ( $<0.05$ vs LPS).

\section{Discussion}

Both conventional therapy and biologically engineered skin substrates are currently used to treat non-healing wounds. Stem cell therapy is a new and promising treatment option. Adipose tissue is a favourable source to harvest MSCs due to the simple procedure and its abundance. ${ }^{16}$ Many methods can be used for cell delivery, such as topical application, local injection and intra-venous infusion. Most of the transplanted cells will be trapped by the capillary vessels in the lung (pulmonary first-pass-effect) after intra-venous infusion. ${ }^{14}$ Topical application is the most commonly used method to transplant cells to the recipient surface. In this study, we used local injection to deliver the MSCs and secretome to the wounds in order to compare the effectiveness of MSC and secretome on wound healing. The transplanted cells were detected 10 days after transplantation, which is comparable with other published works. ${ }^{17}$

Recent studies demonstrated that the paracrine activity plays the critical roles in wound healing. ${ }^{18}$ After injected to the wound surrounding area, most of the MSCs would undergo apoptosis. The remaining cells secreted soluble factors such as VEGF, which accelerated wound healing. In fact, high concentration of VEGF was detected in the secretome used for our study. Therefore, at the early stage, both MSCs and secretome enhanced the wound closure rate, secretome further promoted the process. No significant difference was observed after three weeks, since most of the wound area had healed. Also, more proliferative cells (PCNA positive cells) were observed at the early time points indicating the healing wound process was activated.

MSCs promote the wound healing process by the cell-cell interactions with the wound bed and the modulation of the local microenvironment through the release of chemokines, cytokines and growth factors. ${ }^{19}$ Dermal fibroblast is the major type of dermis, which is responsible for the synthesis and remodelling of extracellular matrix proteins in the proliferative phase after the injury happens. ${ }^{20}$ Its function is closely related to wound closure. In our study, after treated with secretome, the viability, proliferative potential and migration ability were enhanced, which contributed to the improving of wound healing.

Inflammation is also critical for wound healing. Long-term inflammation leads to a refractory ulcer. ${ }^{16}$ The immune-modulation ability has been reported in several studies. After the treatment with MSCs, the 



Figure 3 IHC staining of GFP, VEGF and PCNA. (A) Photo of anti-GFP staining under low magnification (I00x). Two regions were indicated in the image: region a, the wound bed where the MSCs were injected into; region b, the dead tissue covered the wound area (scar). The arrows represented the migrate direction of the injected MSCs: from the lower injected site to the upper area. The red box indicated the area which was showed in the following IHC staining images. (B) Photo of anti-GFP staining at high magnification (200x). The transplanted MSCs were labelled with GFP. The GFP positive cells indicated living MSCs. On day 5 and day 10 after injection, living GFPMSCs were found at the surrounding area of the wound. No living cells were detected after 10 days. (C) IHC staining of VEGF on day 5 and day I4. VEGF was secreted after the injury. Both MSCs and secretome increased the intensity of VEGF, secretome further stimulated the release. (D) IHC staining of PCNA on day 5 and day I4. The positive PCNA signal indicated proliferating cells. Number of PCNA positive cells was higher in the secretome treated tissue. (E): statistical result of anti-PCNA staining. The number of PCNA positive cells was significantly increased after secretome injection on both day 5 and day 14 (day 5 : * $*_{p}<0.05$ vs control, *p < 0.05 vs MSCs; day 14: *p < 0.05 vs control, ${ }^{*} \mathrm{p}<0.05$ vs MSCs). No remarkable difference was observed in MSCs treatment group. 
A

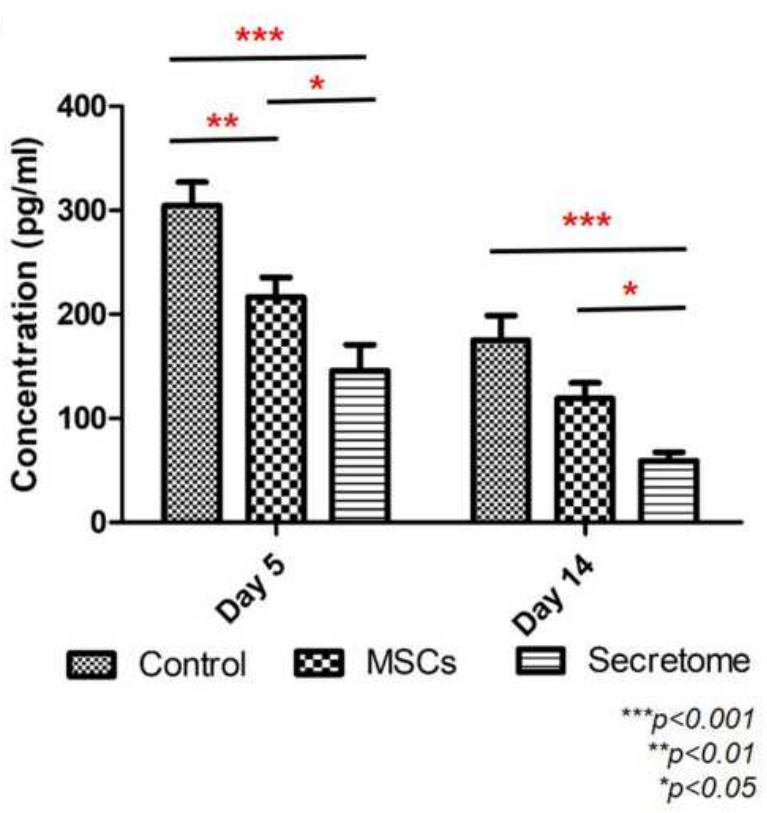

B

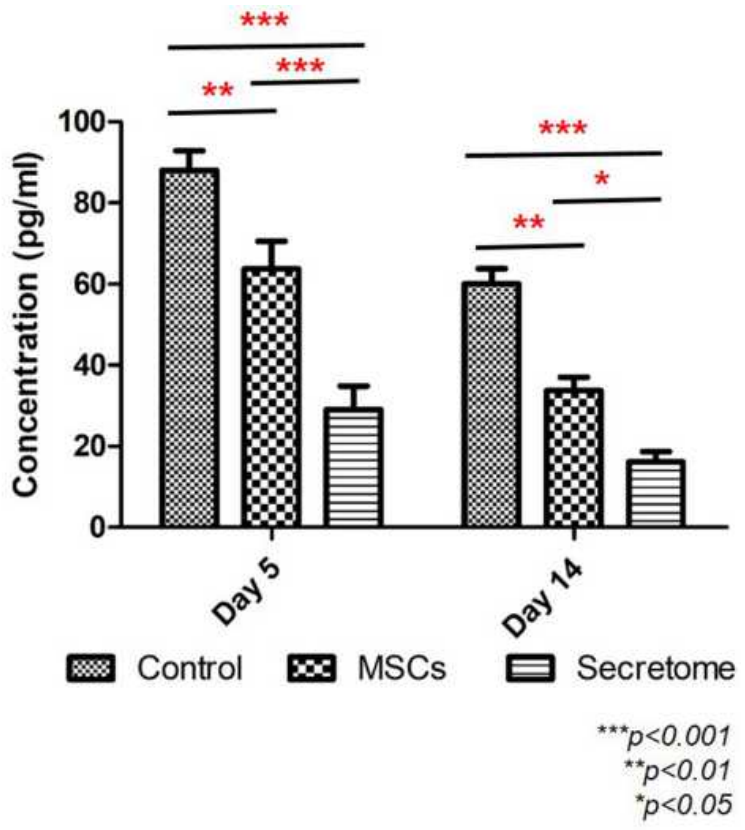

Figure 4 Inflammatory cytokines in blood samples at day 5 and day 14 examined by ELISA. (A) Level of IL-6. The expression of IL- 6 was significantly reduced in day 5 and day 14 after secretome injection (day $5: * * * p<0.001$ vs control, ${ }^{*} p<0.05$ vs MSCs; day 14: ***p $<0.001$ vs control, ${ }^{*} p<0.05$ vs MSCs). The MSCs injection decreased the IL-6 level on day 5 only (**p $<0.01$ ). (B) Level of TNF- $\alpha$. Both MSCs and secretome suppressed the release of TNF- $\alpha$ remarkably (day 5 and day I4: **p $<0.01$ MSCs vs control, ${ }^{* * *} \mathrm{p}<0.001$ secretome vs control). Compared with MSCs, secretome further reduced the secretion (day 5 : $*_{* *} \mathrm{p}<0.001$; day 14: $*_{p}<0.05$ ).

levels of pro-inflammatory cytokines, including IL-1, IL- 6 and TNF- $\alpha$, are reduced; the expressions of antiinflammatory cytokines, such as IL-10 and TSG-6, are up-regulated. $^{21}$ The correlation of IL- 6 and TSG-6 indicates that NF- $\mathrm{NB}$ pathway could be the potential target of MSCs therapy by regulating inflammation. ${ }^{22}$ Inflammation can be modulated through macrophages. After adding secretome into the macrophages, the expression of pro-inflammatory cytokines, including IL-6, TNF- $\alpha$ and MCP-1, was reduced in our study. It was caused by the reduction in pro-inflammatory M1 macrophages and the increase in the anti-inflammatory M2 macrophages. ${ }^{23}$

MSCs promote angiogenesis during wound healing. VEGF is one of the well-known pro-angiogenic factors. It is released by platelets, neutrophils and macrophages, then stimulates the angiogenic process by increasing the vascular permeability of basement membrane and stimulating the endothelial cell migration and proliferation. ${ }^{24}$ In the full-thickness wound, the expression of VEGF reached a peak after 3-7 days. $^{25}$ In our study, the expression of VEGF was examined 5 days after surgery. It demonstrated more VEGF was released after the secretome injection.
Currently, there are around 20 clinical trials using stem cells to treat various types of wound registered on the clinicaltrials.gov website, including second-third degree burn wound, surgical wound, diabetic ulcer, pressure sores and hypertrophic scar. A separate study reported a significant reduction in wound size among the diabetic ulcer patient after treated with autologous adipose derived MSCs. ${ }^{26,27}$

However, there are several limitations in the study. Firstly, the dose was selected according to our previous study, different doses were not tested. Secondly, differentiation of the transplanted cells was not examined. Thirdly, the component of secretome was not fully clear, and more proteins related to wound closure, such as FGF, PDGF and $\mathrm{HGH}$ were not examined.

\section{Conclusion}

Both MSCs and secretome promote wound healing and cell proliferation after local injection. Secretome has the better therapeutic outcome in the early phase due to the promotion in fibroblasts proliferation and migration, together with the suppression in inflammatory response. 


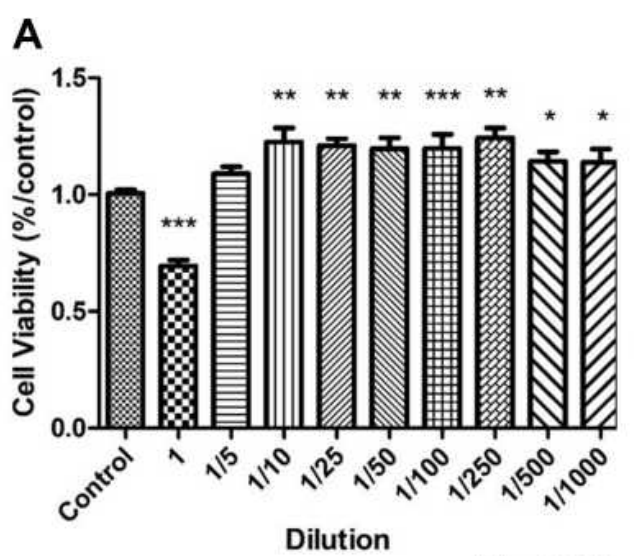

${ }_{* \star *}^{* *} p<0.001$, ${ }^{*} p<0.05$



Dilution

C

control

1 secretome

$1 / 5-1 / 50$ secretome

$\mathrm{Oh}$
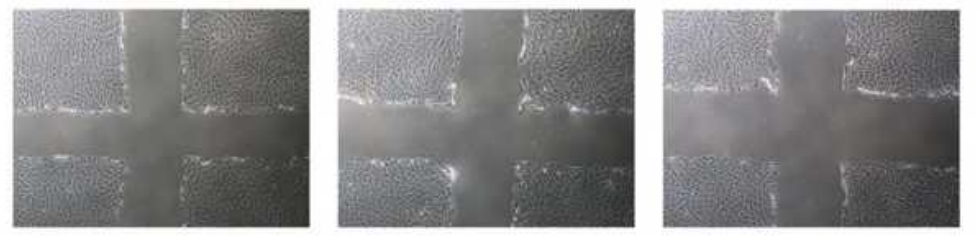

$18 \mathrm{~h}$


D

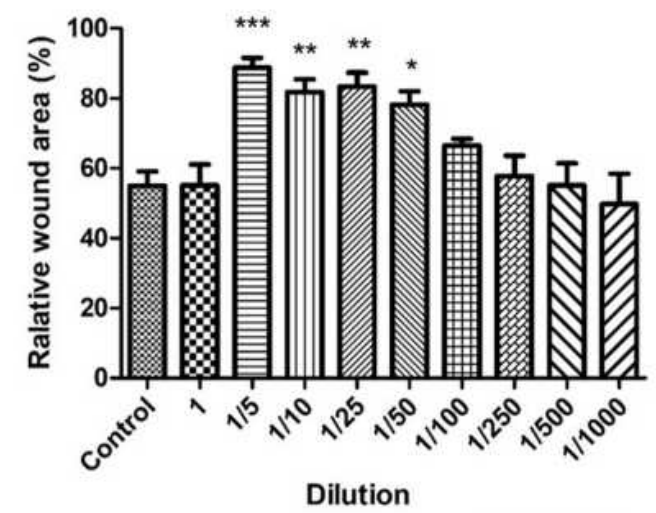

$$
\begin{aligned}
& * * * p<0.001 \\
& * * * 0.01 \\
& * p<0.05
\end{aligned}
$$

Figure 5 The cell viability, proliferation ability and mobility of FR (rat dermal fibroblasts) after treated with secretome. (A) Results of MTT indicated the highest concentration of secretome reduced the metabolic condition of FR $(* * * \mathrm{P}<0.00 \mathrm{I})$. As the concentration decreased from I/I 0 to I/I000, secretome promoted cell viability significantly (I/I0 - I/50, **p < 0.0I; I/I00, ***p < 0.00I; I/250, **p < 0.0I; I/500 - I/I000, *p < 0.05). (B) BrdU proliferation assay indicated the proliferation ability of FR was enhanced when treated with various concentration of secretome $(1 / 5-1 / 25$, ***p $<0.001$; I/50 - I/250, **p < 0.0I; I/500, *p < 0.05). (C) Photos of scratch test taken at $\mathrm{Ch}$ and $18 \mathrm{~h}$. Similar wound area was made at $0 \mathrm{~h}$. After treated with different concentration of secretome for $18 \mathrm{~h}$, the wound was closed at various degrees. The wound treated with secretome showed similar wound area as control (treated with DMEM). When treated with I/5, I/I0, I/25 and I/50 secretome, the wound area was decreased. (D) Statistical results indicated the same finding that the wound area was reduced significantly after treated with I/5, I/I0, I/25 and I/50 secretome $(\mathrm{I} / 5, * * * \mathrm{P}<0.00 \mathrm{I} ; \mathrm{I} / \mathrm{I} 0$, $\left.*^{*} \mathrm{p}<0.01 ; \mathrm{I} / 25, *^{*} \mathrm{p}<0.01 ; \mathrm{I} / 50,{ }^{*} \mathrm{p}<0.05\right)$. 
A
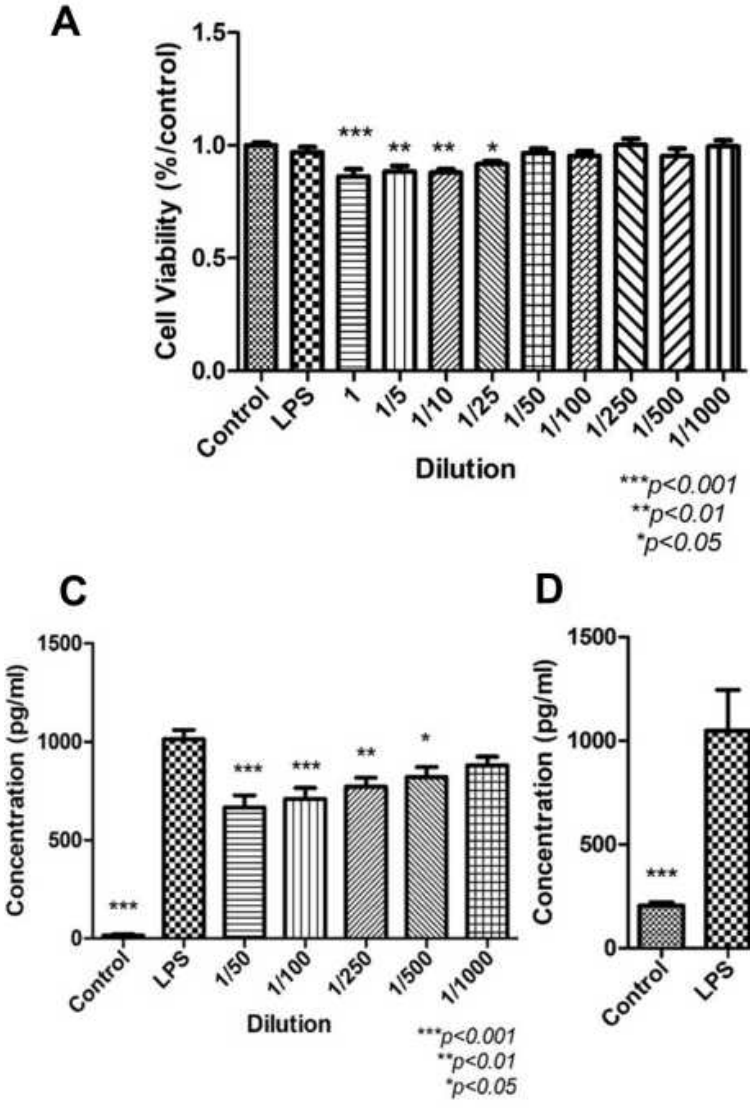

B

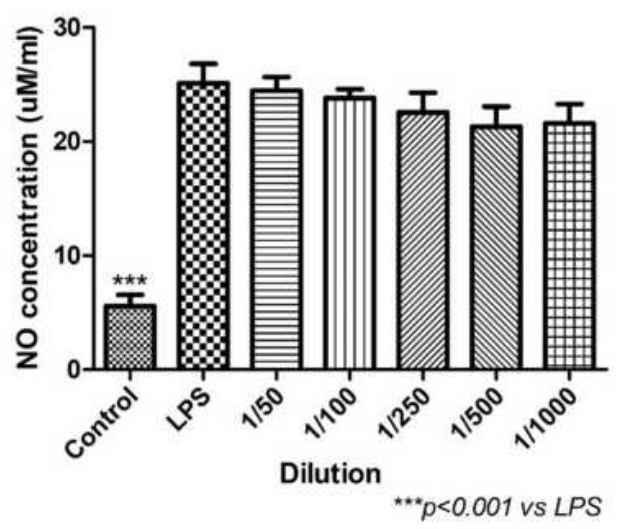

D

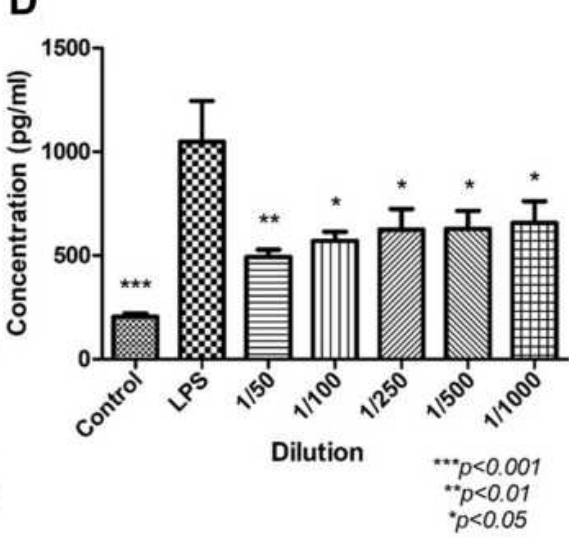

E

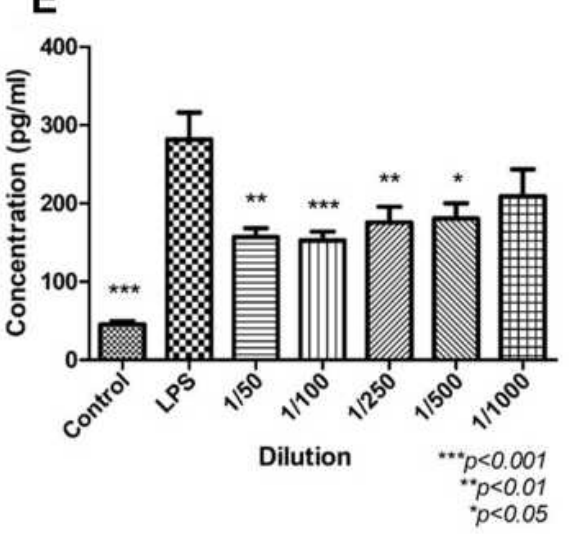

Figure 6 The cell viability, NO assay and ELISA of RAW 264.7 (macrophages) after treated with secretome. (A) The results of MTT indicated that high concentration (I-I/ 25) of secretome suppressed the metabolism of macrophages (I, ***p $<0.00$ I; I/5, **p $<0.0$ I; I/I0, **p $<0.0$ I; I/25, *p $<0.05$ vs control). (B) LPS-induced NO assay. The $\mathrm{NO}$ level was significantly increased after adding LPS into the media $(* * * \mathrm{p}<0.00 \mathrm{I})$. After treated with secretome, no significant reduction was observed. (C-E) ELISA result of pro-inflammatory cytokines IL-6, MCP-I and TNF- $\alpha$. After induced by LPS, the level of pro-inflammatory cytokines was increased significantly $(* * * \mathrm{P}<0.00 \mathrm{I}$ ). After treated with secretome in low concentration, the expressions were down-regulated. IL6: I/50 - I/I00, ***p $<0.001$; I/250, **p $<0.01$; I/500, *p $<0.05$, no significant difference was

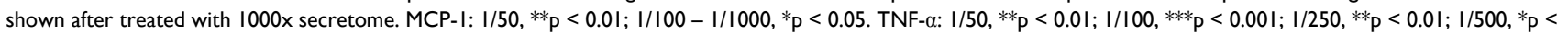
0.05 , no remarkable difference in the supernatant treated with $1 / 1000$ secretome.

\section{Ethical Approval}

The study was approved by the Animal Experimentation Ethics Committee of the Chinese University of Hong Kong.

\section{Statement of Human and Animal Rights}

All procedures were conducted in accordance with the Animals (Control of Experiments) Ordinance Chapter 340, Department of Health, Hong Kong. The study was approved by the Animal Experimentation Ethics Committee of the Chinese University of Hong Kong.

\section{Author Contributions}

All authors made a significant contribution to the work reported, whether that is in the conception, study design, execution, acquisition of data, analysis and interpretation, or in all these areas; took part in drafting, revising or critically reviewing the article; gave final approval of the version to be published; have agreed on the journal to which the article has been submitted; and agree to be accountable for all aspects of the work.

\section{Disclosure}

The authors report no conflicts of interest in this work.

\section{References}

1. Hassan WU, Greiser U, Wang W. Role of adipose-derived stem cells in wound healing. Wound Repair Regen. 2014;22(3):313-325. doi:10.1111/wrr.12173

2. Kanji S, Das H. Advances of stem cell therapeutics in cutaneous wound healing and regeneration. Mediators Inflamm. 2017;2017:5217967. doi:10.1155/2017/5217967

3. Wang PH, Huang BS, Horng HC, Yeh CC, Chen YJ. Wound healing. J Chin Med Assoc. 2018;81(2):94-101. doi:10.1016/j.jcma.2017.11.002 
4. Nussbaum SR, Carter MJ, Fife CE, et al. An economic evaluation of the impact, cost, and medicare policy implications of chronic nonhealing wounds. Value Health. 2018;21(1):27-32. doi:10.1016/j. jval.2017.07.007

5. Sen CK. Human wounds and its burden: an updated compendium of estimates. Adv Wound Care. 2019;8(2):39-48. doi:10.1089/ wound.2019.0946

6. Zarei F, Soleimaninejad M. Role of growth factors and biomaterials in wound healing. Artif Cells Nanomed Biotechnol. 2018;46 (sup1):906-911. doi:10.1080/21691401.2018.1439836

7. Sun BK, Siprashvili Z, Khavari PA. Advances in skin grafting and treatment of cutaneous wounds. Science. 2014;346(6212):941-945. doi:10.1126/science. 1253836

8. Löndahl M, Katzman P, Nilsson A, Hammarlund C. Hyperbaric oxygen therapy facilitates healing of chronic foot ulcers in patients with diabetes. Diabetes Care. 2010;33(5):998-1003. doi:10.2337/ dc09-1754

9. Almalki SG, Agrawal DK. Key transcription factors in the differentiation of mesenchymal stem cells. Differentiation. 2016;92(12):41-51. doi:10.1016/j.diff.2016.02.005

10. Rajabian MH, Ghorabi GH, Geramizadeh B, Sameni S, Ayatollahi M. Evaluation of bone marrow derived mesenchymal stem cells for full-thickness wound healing in comparison to tissue engineered chitosan scaffold in rabbit. Tissue Cell. 2017;49(1):112-121. doi:10.1016/j.tice.2016.11.002

11. Maranda EL, Rodriguez-Menocal L, Badiavas EV. Role of mesenchymal stem cells in dermal repair in burns and diabetic wounds. Curr Stem Cell Res Ther. 2017;12(1):61-70. doi:10.2174/ 1574888X11666160714115926

12. Chang PY, Zhang BY, Cui S, et al. MSC-derived cytokines repair radiation-induced intra-villi microvascular injury. Oncotarget. 2017;8 (50):87821-87836. doi:10.18632/oncotarget.21236

13. Park BS, Jang KA, Sung JH, et al. Adipose-derived stem cells and their secretory factors as a promising therapy for skin aging. Dermatol Surg. 2008;34:1323-1326. doi:10.1111/j.15244725.2008.34283.x

14. Dominici M, Le Blanc K, Mueller I, et al. Minimal criteria for defining multipotent mesenchymal stromal cells. The International Society for Cellular Therapy position statement. Cytotherapy. 2006;8 (4):315-317. doi:10.1080/14653240600855905

15. Ma H, Lam PK, Tong CSW, Lo KKY, Wong GKC, Poon WS. The neuroprotection of hypoxic adipose tissue-derived mesenchymal stem cells in experimental traumatic brain injury. Cell Transplant. 2019;28 (7):874-884. doi:10.1177/0963689719855624

16. Lee DE, Ayoub N, Agrawal DK. Mesenchymal stem cells and cutaneous wound healing: novel methods to increase cell delivery and therapeutic efficacy. Stem Cell Res Ther. 2016;7:37. doi:10.1186/ s13287-016-0303-6
17. Nakamura Y, Ishikawa H, Kawai K, Tabata Y, Suzuki S. Enhanced wound healing by topical administration of mesenchymal stem cells transfected with stromal cell-derived factor-1. Biomaterials. 2013;34 (37):9393-9400. doi:10.1016/j.biomaterials.2013.08.053

18. Tamari M, Nishino Y, Yamamoto N, Ueda M. Acceleration of wound healing with stem cell-derived growth factors. Int J Oral Maxillofac Implants. 2013;28(6):e369-e375. doi:10.11607/jomi.te17

19. Hassanshahi A, Hassanshahi M, Khabbazi S, et al. Adipose-derived stem cells for wound healing. J Cell Physiol. 2019;234 (6):7903-7914. doi:10.1002/jcp.27922

20. Stunova A, Vistejnova L. Dermal fibroblasts-A heterogeneous population with regulatory function in wound healing. Cytokine Growth Factor Rev. 2018;39:137-150. doi:10.1016/j.cytogfr.2018.01.003

21. Liu L, Yu Y, Hou Y, et al. Human umbilical cord mesenchymal stem cells transplantation promotes cutaneous wound healing of severe burned rats. PLoS One. 2014;9(2):e88348. doi:10.1371/journal. pone. 0088348

22. Zhang R, Liu Y, Yan K, et al. Anti-inflammatory and immunomodulatory mechanisms of mesenchymal stem cell transplantation in experimental traumatic brain injury. $J$ Neuroinflammation. 2013;10:106. doi:10.1186/1742-2094-10-106

23. Zhang QZ, Su WR, Shi SH, et al. Human gingiva-derived mesenchymal stem cells elicit polarization of $\mathrm{m} 2$ macrophages and enhance cutaneous wound healing. Stem Cells. 2010;28(10):1856-1868. doi:10.1002/stem.503

24. Bao P, Kodra A, Tomic-Canic M, Golinko MS, Ehrlich HP, Brem H. The role of vascular endothelial growth factor in wound healing. J Surg Res. 2009;153(2):347-358. doi:10.1016/j.jss.2008.04.023

25. Frank S, Hübner G, Breier G, Longaker MT, Greenhalgh DG, Werner S. Regulation of vascular endothelial growth factor expression in cultured keratinocytes. Implications for normal and impaired wound healing. $J$ Biol Chem. 1995;270(21):12607-12613. doi:10.1074/jbc.270.21.12607

26. Lam PK, Lo W, Tong CS, Lo KY, Chook P, Leung PC. Treatment of chronic non-healing ulcers with adipose tissue-derived mesenchymal stem cells - a pilot study. Cytotherapy. 2018;20(5):S45. doi:10.1016/ j.jcyt.2018.02.116

27. Lam PK, Lo W, Tong CS, et al. Topical application of adiposederived mesenchymal stem cells in the treatment of a non-healing ulcer in a leper: a case report - a new option for treatment of extremely chronic ulcer. World J Surg and Surg Res. 2018;1:1.
Clinical, Cosmetic and Investigational Dermatology is an international, peer-reviewed, open access, online journal that focuses on the latest clinical and experimental research in all aspects of skin disease and cosmetic interventions. This journal is indexed on CAS.
The manuscript management system is completely online and includes a very quick and fair peer-review system, which is all easy to use. Visit http://www.dovepress.com/testimonials.php to read real quotes from published authors. 